\title{
Transition temperature from band to hopping direct current conduction in crystalline semiconductors with hydrogen-like impurities: Heat versus Coulomb attraction
}

\author{
N. A. Poklonski, ${ }^{1, a)}$ S. A. Vyrko, ${ }^{1}$ O. N. Poklonskaya, ${ }^{1}$ and A. G. Zabrodskii ${ }^{2}$ \\ ${ }^{1}$ Belarusian State University, pr. Nezavisimosti 4, Minsk 220030, Belarus \\ ${ }^{2}$ Ioffe Physicotechnical Institute RAS, Politekhnicheskaya ul. 26, St. Petersburg 194021, Russia
}

(Received 9 September 2011; accepted 28 October 2011; published online 19 December 2011)

\begin{abstract}
For nondegenerate bulk semiconductors, we have used the virial theorem to derive an expression for the temperature $T_{\mathrm{j}}$ of the transition from the regime of "free" motion of electrons in the $c$-band (or holes in the $v$-band) to their hopping motion between donors (or acceptors). Distribution of impurities over the crystal was assumed to be of the Poisson type, while distribution of their energy levels was assumed to be of the Gaussian type. Our conception of the virial theorem implementation is that the transition from the band-like conduction to hopping conduction occurs when the average kinetic energy of an electron in the $c$-band (hole in the $v$-band) is equal to the half of the absolute value of the average energy of the Coulomb interaction of an electron (hole) with the nearest neighbor ionized donor (acceptor). Calculations of $T_{\mathrm{j}}$ according to our model agree with experimental data for crystals of $\mathrm{Ge}, \mathrm{Si}$, diamond, etc. up to the concentrations of a hydrogen-like impurity, at which the phase insulator-metal transition (Mott transition) occurs. Under the temperature $T_{\mathrm{h}} \approx T_{\mathrm{j}} / 3$, when the nearest neighbor hopping conduction via impurity atoms dominates, we obtained expressions for the electrostatic field screening length $\Lambda_{\mathrm{h}}$ in the Debye-Hückel approximation, taking into account a nonzero width of the impurity energy band. It is shown that the measurements of quasistatic capacitance of the semiconductor in a metal-insulator-semiconductor structure in the regime of the flat bands at the temperature $T_{\mathrm{h}}$ allow to determine the concentration of doping impurity or its compensation ratio by knowing $\Lambda_{\mathrm{h}}$. C 2011 American Institute of Physics. [doi:10.1063/1.3667287]
\end{abstract}

\section{INTRODUCTION}

In order to form highly sensitive photodetectors (from infrared to terahertz wavelengths) based on crystalline semiconductors, the hopping conduction via hydrogen-like impurities should be inhibited (see, e.g., Refs. 1 and 2). It is necessary to block (minimize the value of) hopping conduction by epitaxially growing the layer of semiconductor with a low content of impurities at the surface of the heavily doped semiconductor; this technique provides the energetic barrier for electron (hole) transport over the impurity band states. For this purpose, it is useful to know the characteristic temperature $T_{\mathrm{j}}$ (see Fig. 1), when the band-like electrical conduction (of electrons in the $c$-band or holes in the $v$-band) is superseded by the hopping conduction via impurities. Our work aims to present a quasiclassical model of the temperature $T_{\mathrm{j}}$ dependence on the concentration of hydrogenlike impurities in crystalline semiconductors.

In homogeneous-doped and nondegenerate semiconductors, drift and diffusion of electrons and/or holes are commonly described in the terms of their transitions between singleelectron states (see, e.g., Refs. 3-8). The direct current electrical conduction is tentatively subdivided into three regimes (mechanisms) (see Fig. 1): the propagating (or band) conduction (BC), the hopping conduction (HC), and the hybrid regime $(\mathrm{BC}+\mathrm{HC})$, which is sometimes called the jumping conduction. ${ }^{4}$ It should be noted that, at the characteristic temperature

${ }^{a)}$ Electronic mail: poklonski@bsu.by.
$T_{\mathrm{j}}$, the electrical conductivity $\sigma_{p}=1 / \rho_{p}$ with thermal activation energy $\varepsilon_{1}$ in the $\mathrm{BC}$ regime is equal to the electrical conductivity $\sigma_{\mathrm{h}}=1 / \rho_{\mathrm{h}}$ with thermal activation energy $\varepsilon_{3}$ in the nearest neighbor hopping $(\mathrm{NNH})$ regime of the dc conduction. Here, resistivity in the $\mathrm{BC}$ regime is $\rho_{p}=\rho_{1} \exp \left(\varepsilon_{1} / k_{\mathrm{B}} T\right)$ and resistivity in the $\mathrm{NNH}$ regime is $\rho_{\mathrm{h}}=\rho_{3} \exp \left(\varepsilon_{3} / k_{\mathrm{B}} T\right)$, where $k_{\mathrm{B}} T$ is the thermal energy and $\rho_{1}, \varepsilon_{1}, \rho_{3}$, and $\varepsilon_{3}$ are the parameters which weakly depend on temperature $T$. Some partial expressions for $T_{\mathrm{j}}$ obtained by fitting experimental data for germanium crystals of $p$-type are reported in Refs. 9 and 10. However, for applications, it is necessary to have an analytical dependence of $T_{\mathrm{j}}$ on concentration $N$ of the majority (doping) impurity and the ratio $0<K<1$ of its compensation by the minority impurity with the concentration $K N$.

The $\mathrm{BC}$ regime is realized in crystalline semiconductors at temperatures $T>T_{\mathrm{j}}$. Here, the states of electrons in the $c$-band and the states of holes in the $v$-band are characterized by eigenvalues of a quasimomentum operator and average values of lifetime in delocalized states of the $c$ - or $v$-bands, which are determined by phonons and atomic defects of a lattice (as well as by a capture of electrons and holes on traps and an electronhole recombination). In the $\mathrm{BC}$ regime, electrons and holes are "free" to move by tunneling in the crystalline matrix between successive acts of scattering on structure defects and phonons. In the $\mathrm{HC}$ regime, an ordinary mobile electron (or hole) is localized near a single impurity atom the majority of the time. Electrons (or holes) can migrate between immovable impurities only due to phonons (or other quasiparticles); thus, the conductivity is exponentially decreased under the temperature 


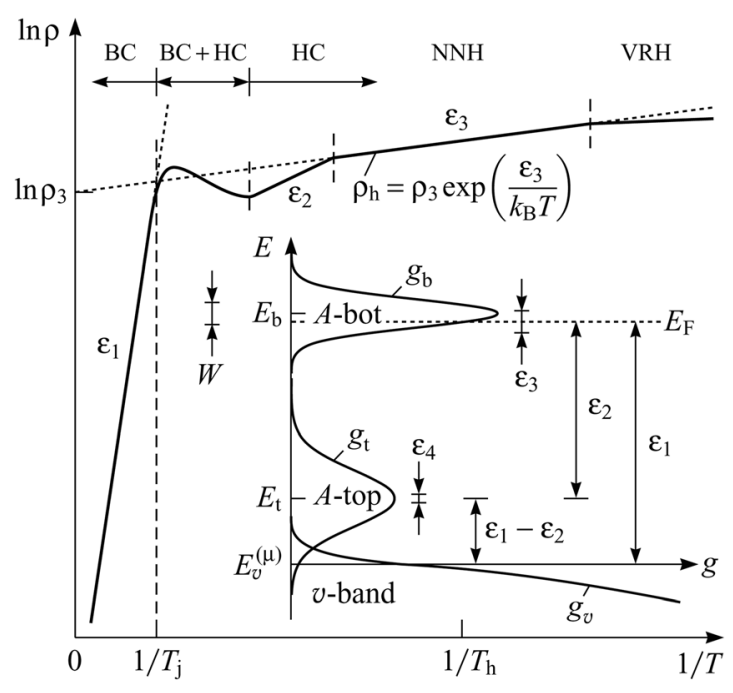

FIG. 1. Schematic inverse temperature $(1 / T)$ dependence of logarithm of the electrical resistance $(\ln \rho)$ of $p$-type crystalline semiconductor in different regimes of hole transport: $\mathrm{BC}$ is the band conduction, $\mathrm{BC}+\mathrm{HC}$ is the mixture of the band and hopping conductions, $\mathrm{HC}$ is the hopping conduction, $\mathrm{NNH}$ is the hopping conduction $\sigma_{\mathrm{h}}=1 / \rho_{\mathrm{h}}$ via the nearest neighbor acceptors, and VRH is the hopping conduction via nearest acceptors by energy. In the inset: $g_{v}$ is the density of states near the top of the valence band ( $E$ is the single-electron energy, $E_{v}^{(\mu)}$ is the mobility edge of holes in the $v$-band), $g_{\mathrm{b}}$ and $g_{\mathrm{t}}$ are the density of states of the $A$-bot and the $A$-top bands (centered on $E_{\mathrm{b}}$ and $\left.E_{\mathrm{t}}\right)$ of a hydrogen-like acceptor impurity, which can be in three charge states $(-1,0,+1)$ (see also Ref. 3 ). (In compensated semiconductors $(K>0.1)$ the $A$-top band is not pronounced and acceptors can be only in the charge states ( 0$)$ and $(-1)$ of the $A$-bot band. $D$-bot and $D$-top bands of hydrogen-like donors are below the mobility edge of electrons in the $c$-band and are not shown.)

decrease. In the $\mathrm{BC}+\mathrm{HC}$ regime, ${ }^{4}$ electrons (or holes) are almost delocalized, but the phase coherence is destroyed for their thermally activated tunneling motion from atom to atom (it can be an atom of a crystalline matrix as well as an impurity atom). It should be noted that, in the band scheme (see the inset in Fig. 1), the $\mathrm{BC}+\mathrm{HC}$ regime corresponds to merged-intoeach-other conduction mechanisms: the thermally activated tunneling of holes in the $A$-top band (activation energy $\varepsilon_{4}$ ) and hole transitions from the $A$-top band to the $v$-band (activation energy $\left.\varepsilon_{1}-\varepsilon_{2}\right)$. On the one hand, in contrast to the $\mathrm{BC}$ regime, alternating paths of hole tunneling have accidental phases and do not interfere with each other. On the other hand, in contrast to the $\mathrm{HC}$ regime with thermal activation energies $\varepsilon_{2}$ and $\varepsilon_{3}$ (in the temperature range close to $T_{\mathrm{h}} \approx T_{\mathrm{j}} / 3$ ), thermally activated tunneling in the $\mathrm{BC}+\mathrm{HC}$ regime slightly depends on temperature comparing to the hopping conduction (activation energy $\varepsilon_{4}<\varepsilon_{3}<\varepsilon_{2}$ ). [Note that the temperature $T_{\mathrm{h}} \approx T_{\mathrm{j}} / 3$ was chosen as an average value from analysis of dependences $\ln (\rho)$ on $1 / T$ in the $\mathrm{NNH}$ temperature range of whole experimental data from Refs. 9, 11-31.] The activation energy $\varepsilon_{1}$ corresponds to the transition of a typical acceptor from the charge state $(0)$ to the charge state $(-1)$ and appearance of a hole in the $v$-band. The activation energy $\varepsilon_{2}$ corresponds to the transition of two electrically neutral acceptors into negative- and positivecharged ions; this transition is contributed to the $\varepsilon_{2}$-conduction. The activation energy $\varepsilon_{1}-\varepsilon_{2}<\varepsilon_{2}$ corresponds to the transition of a typical acceptor from the charge state $(+1)$ to the charge state $(0)$ and appearance of a hole in the $v$-band. As a rule, in the $\mathrm{BC}+\mathrm{HC}$ regime, it is quite difficult to measure thermal activation energies $\varepsilon_{4}$ and $\varepsilon_{1}-\varepsilon_{2}$, which are not pronounced in the galvanomagnetic measurements and require the lowtemperature photoconduction measurements. ${ }^{32}$

For a $p$-type semiconductor at very low temperatures (less than $T_{\mathrm{h}} / 2$ ), conduction is realized by hops of holes (electron vacancies) between acceptors which are not necessarily neighbored to each other, but which have a minimal difference in their energy levels. [We estimate that the variable range hopping (VRH) regime starts dominating over the NNH regime at a temperature less than $T_{\mathrm{h}} / 2$, which was chosen as an average value from analysis of dependences $\ln \left(\rho_{\mathrm{h}}\right)$ on $1 / T$ (see Fig. 1) of experimental data from Refs. 9, 11-31. In the temperature range $T<T_{\mathrm{h}} / 2$, the hopping activation energy $\varepsilon_{3}$ behaves as $\varepsilon_{3} \propto T^{3 / 4}$ (Mott model) $^{33}$ or $\varepsilon_{3} \propto T^{1 / 2}$ (EfrosShklovskii model).$^{34}$ ] This regime is usually called variable range hopping (VRH). When the temperature increases, this mechanism of conduction is superseded by hops of holes between the nearest neighbor acceptors (NNH) with activation energy $\varepsilon_{3}$, which is equal within an order of magnitude to the root-mean-square fluctuation $W$ of their energy levels (the width of the acceptor $A$-bot band). [In Ref. 35, a relation of proportionality for boundary temperature between $\mathrm{NNH}$ and VRH regimes is obtained based on statistics of pair donors, between which an electron jump takes place, taking into account the $D$-bot and $D$-top donor bands in crystalline $n$-type semiconductors.] Further increase of temperature leads to the emission of the holes from the $A$-bot band to the $A$-top band; $\varepsilon_{2}$-conduction takes place. In the temperature region intermediate between $\varepsilon_{2}$-conduction and $\varepsilon_{1}$-conduction, the $\mathrm{BC}+\mathrm{HC}$ regime can be observed. For temperatures $T>T_{\mathrm{j}}$, the emission of holes from the $A$-bot and $A$-top bands to the $v$-band occurs as well as the capture of holes from the $v$-band on the $A$-bot and $A$-top bands. These processes correspond to the band conduction $(\mathrm{BC})$ with activation energy $\varepsilon_{1}$, which is equal within an order of magnitude to the thermal ionization energy of the acceptor. According to the common experimental observations, the conduction with activation energy $\varepsilon_{2}$ rarely happens in crystalline semiconductors with hydrogen-like impurities for compensation ratios $K>0.1{ }^{36}$ Note that $\varepsilon_{2}$ energy can be considered as a difference between energies of the ground and excited states of a neutral acceptor, e.g., a boron atom in the diamond crystalline matrix site. ${ }^{37}$ In the $\mathrm{BC}+\mathrm{HC}$ regime $\left(T<T_{\mathrm{j}}\right)$, the decrease of resistivity $\rho=1 / \sigma$ with decreasing in $T$ is caused by capturing holes from the $v$-band on acceptors in the charge state $(-1)$ and by the consequent decrease of scattering on ions for the holes left in the $v$-band. It is clear that, for all three regimes of hole transport $(\mathrm{BC}, \mathrm{BC}+\mathrm{HC}$, and $\mathrm{HC})$, the charge states $(0)$ and $(-1)$ of immobile acceptors can migrate over a crystal and all donors, which are in the charge state $(+1)$, are also immobile. Charge state migration occurs due to the capture of holes from the $v$-band on acceptors and emission of holes from acceptors into the $v$-band and also due to hops of holes from acceptors in the charge state (0) to acceptors in the charge state $(-1)$. Figure 1 shows (not in the scale) a typical picture in moderately doped and moderately compensated semiconductors, when only the $\mathrm{BC}$ and $\mathrm{HC}$ regimes are well approximated by straight lines in the dependence of $\ln \rho$ on $1 / T$. 


\section{CALCULATIONS OF TRANSITION TEMPERATURE FROM THE BAND TO HOPPING CONDUCTION}

Let us consider a nondegenerate semiconductor of $p$-type, in which only the $A$-bot acceptor band is pronounced. We have adopted that $p$ is the concentration of holes in the $v$ band (valence band), $N=N_{0}+N_{-1}$ is the total concentration of acceptors in the charge states $(0)$ and $(-1)$, and $K N$ is the concentration of donors, which are all in the charge state $(+1)$. Thus, an electroneutrality condition of semiconductor has the form: $p=N_{-1}-K N$. The band mechanism of the conduction is determined by the motion of gas of holes, and it is characterized by the average value of kinetic energy of a hole, which is equal to $3 k_{\mathrm{B}} T / 2$, where $k_{\mathrm{B}}$ is the Boltzmann constant and $T$ is the absolute temperature. Let us note that, at $T \approx T_{\mathrm{j}}$ (Fig. 1), the concentration of holes in the $v$-band $p \ll K(1-K) N$, so we yield $N_{-1}=N_{+1}=K N$, where $N_{-1}$ and $N_{+1}$ are concentrations of ionized acceptors and donors (see, e.g., Ref. 38). In accordance with models (Refs. 39 and 40), the effective concentration of holes hopping between acceptors is $N_{\mathrm{h}}=N_{0} N_{-1} / N$; thus, from the condition $\sigma_{p}=\sigma_{\mathrm{h}}$, i.e., $e p \mu_{p}=e N_{\mathrm{h}} M_{\mathrm{h}}$, at temperature $T_{\mathrm{j}}$, it follows that $p \ll N_{\mathrm{h}}$ and the drift mobility of holes in the $v$-band $\mu_{p}$ is much larger than the drift hopping mobility of holes $\mathrm{M}_{\mathrm{h}}$ via acceptors.

If particles in an equilibrium system interact obeying the Coulomb's law, then according to the virial theorem (see, e.g., Ref. 41 and 42),

$$
E_{\mathrm{kin}}=-\frac{1}{2} U_{\mathrm{tot}},
$$

where $E_{\text {kin }}$ is the average kinetic energy of all particles and $U_{\text {tot }}$ is the average potential energy of the Coulomb interaction between all particles. In a first order approximation, the virial theorem can be applied separately to two subsystems (see, e.g., Ref. 42): i) holes and migrating over a crystal the charge states $(-1)$ of immobile acceptors and ii) immobile donors in the charge state $(+1)$, electrically neutral acceptors (bound states of holes and negatively charged acceptors), and bounded electrons and nuclei of the crystalline matrix. Further, for calculations of $T_{\mathrm{j}}$, we apply the virial theorem to the first subsystem.

The virial theorem is applicable to the semiconductor at any temperature. At temperatures $T>T_{\mathrm{j}}$, the $v$-band conduction dominates and holes freely move over the crystal. Then, the average kinetic energy of a typical hole in the $v$-band is equal to $3 k_{\mathrm{B}} T / 2$; however, the average potential energy of the hole is not known. At temperatures $T<T_{\mathrm{j}}$, the hopping conduction dominates and holes in the $A$-bot band are quasilocalized on acceptors. Then, the hole potential energy is equal to the Coulomb energy of interaction between a hole and the nearest acceptor, whereas the average kinetic energy of a typical hole moving by means of hops is not known. Thus, only at the edge between hopping and band conductions, we can assess both the average kinetic energy and the average potential energy of a hole in the field of the negatively charged acceptor. Since they are connected to each other according to the virial theorem, the temperature in the kinetic energy gives the temperature of equality between the hopping and band conductions. To this end, the transition from the band conduction to the hopping conduction at $T=T_{\mathrm{j}}$ is determined by equality of the average kinetic energy of motion of a free (delocalized) hole in the v-band $E_{\mathrm{kin}} \rightarrow 3 k_{\mathrm{B}} T_{\mathrm{j}} / 2$ to the half of the average potential energy of a hole in the Coulomb field of the nearest ionized acceptor

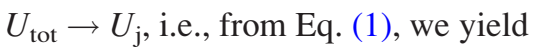

$$
\frac{3 k_{\mathrm{B}} T_{\mathrm{j}}}{2}=-\frac{U_{\mathrm{j}}}{2} .
$$

We take into account that all the other holes of the $v$-band, acceptors in the charge states $(-1)$ and $(0)$, and donors in the charge state $(+1)$ by virtue of the electrical neutrality of a crystal form an electrically neutral background. It is clear that, at temperature $T_{\mathrm{j}}$, due to low concentration of holes, the interhole interaction can be neglected as well as the possibility of capturing holes on acceptors in the charge state (0). These assumptions allow to approximately describe the many-particle closed system with the pair Coulomb interaction between charged particles as a set of two-particle systems containing a hole and the closest to it acceptor in the charge state $(-1)$. Note that the proposed procedure of the virial theorem application in description of the interaction of charge carriers and impurity ions in nondegenerate semiconductor resembles a scheme of substantiation of the Boltzmann kinetic equation in Ref. 43.

In order to determine $U_{\mathrm{j}}$, we assume that acceptors in the charge states $(-1)$ and $(0)$ and donors in the charge state $(+1)$ are randomly distributed in a crystal, i.e., according to the Poisson distribution. Then, the probability that, at the distance $r$ from hole in the interval $(r, r+\mathrm{d} r)$, the nearest negatively charged acceptor occurs and there are no negatively charged acceptors in the volume $4 \pi r^{3} / 3$ is (see, e.g., Refs. 44 and 45 )

$$
\mathcal{P}(r) \mathrm{d} r=4 \pi r^{2} K N \exp \left(-\frac{4 \pi}{3} r^{3} K N\right) \mathrm{d} r,
$$

where $K N$ is the concentration of ionized acceptors, i.e., acceptors in the charge state $(-1)$.

The average Coulomb energy of the hole interaction with the nearest negatively charged acceptor, taking into account Eq. (3), is

$$
\begin{aligned}
U_{\mathrm{j}} & =\int_{0}^{\infty} U(r) \mathcal{P}(r) \mathrm{d} r=-\Gamma\left(\frac{2}{3}\right) \frac{e^{2}}{4 \pi \varepsilon_{\mathrm{r}} \varepsilon_{0}}\left(\frac{4 \pi}{3} K N\right)^{1 / 3} \\
& \approx-1.354 \frac{e^{2}}{4 \pi \varepsilon_{\mathrm{r}} \varepsilon_{0}}\left(\frac{4 \pi}{3} K N\right)^{1 / 3},
\end{aligned}
$$

where $U(r)=-e^{2} /\left(4 \pi \varepsilon_{\mathrm{r}} \varepsilon_{0} r\right)$ is the potential energy of a $v$ band hole interacting by the Coulomb's law with the nearest neighbor acceptor in the charge state $(-1), e$ is the elementary charge, $\varepsilon_{\mathrm{r}}$ is the relative permittivity of the crystalline matrix, $\varepsilon_{0}$ is the electric constant, $\Gamma(z)=\int_{0}^{\infty} t^{z-1} \exp (-t) \mathrm{d} t$ is the Euler's gamma-function, and $N_{-1}=K N$ is the concentration of negatively charged acceptors.

Thus, substitution of expression (4) into Eq. (2) yields

$$
k_{\mathrm{B}} T_{\mathrm{j}} \approx 0.73 \frac{e^{2}}{4 \pi \varepsilon_{\mathrm{r}} \varepsilon_{0}}(K N)^{1 / 3},
$$


where $T_{\mathrm{j}}$ is the temperature of equality of hopping and band conductions (Fig. 1). [For description of kinetic phenomena in gas plasma, the Landau length $d_{\mathrm{L}}=e^{2} /\left(4 \pi \varepsilon_{\mathrm{r}} \varepsilon_{0} k_{\mathrm{B}} T\right)$ is used. ${ }^{46,47}$ At the temperature $T=T_{\mathrm{j}}$ for a nondegenerate gas of $v$-band holes against a background of neutralizing their charge negatively charged acceptors from Eq. (5), we obtain $d_{\mathrm{L}} \approx 1.37(K N)^{-1 / 3}$.]

Let us now relate the temperature $T_{\mathrm{j}}$ with the ionization energy of a single hydrogen-like impurity $I_{\text {im }}$ and its concentration $N_{\mathrm{M}}$, which corresponds to the phase insulator-metal transition (the Mott transition) ${ }^{48}$.

The concentration of majority (doping) hydrogen-like impurity $N=N_{\mathrm{M}}$, which corresponds to the Mott transition, and the Bohr radius $a_{\mathrm{im}}$ of a single (insulated) doping impurity in a crystal are satisfied by the relation ${ }^{49}$

$$
N_{\mathrm{M}}^{1 / 3} a_{\mathrm{im}} \approx \frac{0.542}{\left[(1-K)\left(\varepsilon_{\mathrm{r}}+2\right)\right]^{1 / 3}} .
$$

Let us divide the left and right parts of the relation (5) by ionization energy of the hydrogen-like impurity $I_{\text {im }}=$ $e^{2} /\left(8 \pi \varepsilon_{\mathrm{r}} \varepsilon_{0} a_{\mathrm{im}}\right)$ and then multiply and divide the right part of the obtained expression by $N_{\mathrm{M}}{ }^{1 / 3}$; thus, from Eq. (5), taking into account Eq. (6), we obtain

$$
\frac{k_{\mathrm{B}} T_{\mathrm{j}}}{I_{\mathrm{im}}} \approx 0.79\left[\frac{K}{(1-K)\left(\varepsilon_{\mathrm{r}}+2\right)}\right]^{1 / 3}\left(\frac{N}{N_{\mathrm{M}}}\right)^{1 / 3} .
$$

Calculations by using Eq. (7) were compared with experimental data for $p$-Ge crystals doped with gallium (and compensated with arsenic and partially with selenium) by transmutation reactions under the influence of thermal neutrons of a nuclear reactor. ${ }^{50,51}$ Figure 2 shows $T_{\mathrm{j}}$ values (points $a-f$ ), which we obtain according to Fig. 1 from the experimental dependences of $\ln \rho$ on $1 / T$ of germanium samples (Refs. 9 and 12-17). Experimental values of $T_{\mathrm{j}}$ were

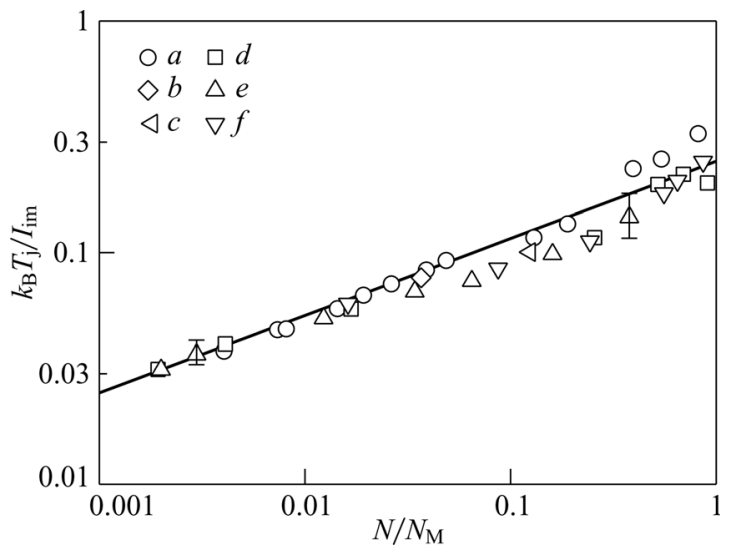

FIG. 2. Dependence on concentration $N$ of gallium atoms of the transition temperature $T_{\mathrm{j}}$ from hopping to the band conduction in neutron-transmutation-doped $p$-Ge:Ga crystals. The line is the calculations by using Eq. (7) for $K=0.35$. The points $a-f$ are experimental data from the works: ( $a$ ) Refs. 12 and 13, (b) Ref. 14, (c) Ref. 15, (d) Ref. 9, (e) Ref. 16, and ( $f$ ) Ref. 17. The typical deviation of $T_{\mathrm{j}}$ for two values of $N$ (at the beginning and at the end of doping degree range) is indicated by vertical bars.

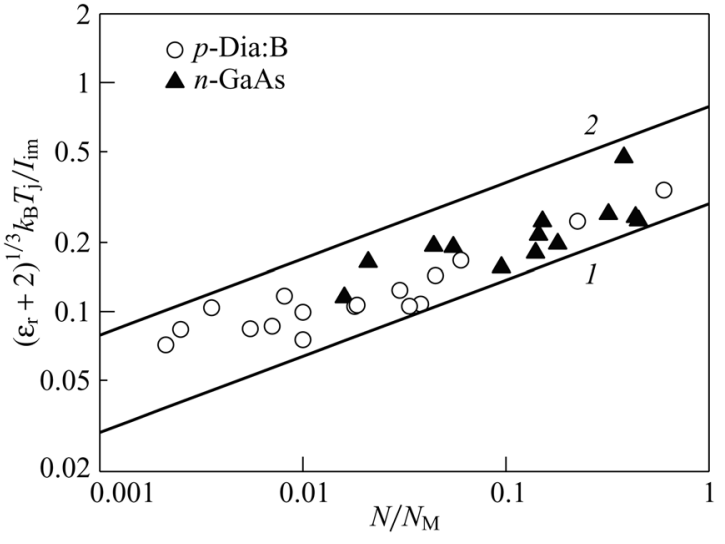

FIG. 3. Dependence on concentration of the doping impurity $N$ of the transition temperature $T_{\mathrm{j}}$ from hopping to the band conduction in $p$-Dia:B and $n$-GaAs. The lines are calculations by using Eq. (7) for $K=0.05$ (1) and $K=0.5$ (2). The points are experimental data from the works: Refs. 18-24 ( $p$-Dia:B) and Refs. 25-27 (n-GaAs).

obtained as an intersection between the straight lines, representing the asymptotic behaviors of the band and hopping conductions $\ln \rho_{p}=\ln \rho_{1}+\varepsilon_{1} / k_{\mathrm{B}} T$ and $\ln \rho_{\mathrm{h}}=\ln \rho_{3}+\varepsilon_{3} / k_{\mathrm{B}} T$, respectively, assuming $\rho_{1}, \rho_{3}, \varepsilon_{1}$, and $\varepsilon_{3}$ to be constants (Fig. 1). The deviations of the experimental data from the fitting straight lines were within $3 \%$.

The line in Fig. 2 shows the calculations of $T_{\mathrm{j}}$ using Eq. (7) for the compensation ratio $K=0.35$. Parameters of $p$-Ge:Ga are presented in Table I. ${ }^{17,23,52-59}$ It is seen that the temperature $T_{\mathrm{j}}$ is described in the wide range of doping impurity concentration by relation (7). The small deviations of $T_{\mathrm{j}}$ from the calculations are due to the fact that the neutrontransmutation doping of germanium crystals allows accurately, within percents, to control the concentration of the doping impurity $(\mathrm{Ga})$ and the compensation ratio of samples (see, e.g., Refs. 50, 51, 60, and 61).

Figure 3 shows the dependence of the transition temperature $T_{\mathrm{j}}$ from hopping to the band conduction in boron-doped

TABLE I. Semiconductor crystal parameters.

\begin{tabular}{lccc}
\hline \hline & $\varepsilon_{\mathrm{r}}^{\mathrm{a}}$ & $I_{\mathrm{im}}, \mathrm{meV}^{\mathrm{a}}$ & $N_{\mathrm{M}}, \mathrm{cm}^{-3}$ \\
\hline$p$-Ge:Ga & 15.4 & 11.32 & $1.85 \times 10^{17 \mathrm{~b}}$ \\
$p$-Dia:B & 5.7 & 370 & $2 \times 10^{20 \mathrm{c}}$ \\
$n$-GaAs & 12.4 & 6 & $2 \times 10^{16 \mathrm{~d}}$ \\
$n$-Si:Sb & 11.5 & 42.7 & $3 \times 10^{18 \mathrm{e}}$ \\
$n$-Si:P & 11.5 & 45.6 & $\approx 4 \times 10^{18 \mathrm{f}}$ \\
$n$-Si:As & 11.5 & 53.8 & $7.8 \times 10^{18 \mathrm{~g}}$ \\
$p$-Si:B & 11.5 & 44.39 & $4 \times 10^{18 \mathrm{~h}}$ \\
$p$-Si:Al & 11.5 & 69.03 & $1.6 \times 10^{19 \mathrm{i}}$ \\
$p$-Si:Ga & 11.5 & 72.73 & $4.7 \times 10^{17 \mathrm{i}}$ \\
$n$-ZnSe:Al & 8.6 & 23.6 & \\
\hline \hline
\end{tabular}

${ }^{\mathrm{a}}$ Data on $\varepsilon_{\mathrm{r}}$ and $I_{\mathrm{im}}$ are taken from Ref. 52.

${ }^{\mathrm{b}}$ Reference 17 .

${ }^{\mathrm{c}}$ Reference 23

${ }^{\mathrm{d}}$ References 53 and 54.

${ }^{\mathrm{e}}$ Reference 55.

${ }^{\mathrm{f}}$ References 56 and 57.

${ }^{\mathrm{g}}$ Reference 58.

${ }^{\mathrm{h}}$ Reference 59 .

i The Mott concentration of impurity $N_{\mathrm{M}}$ is assessed by Eq. (6). 


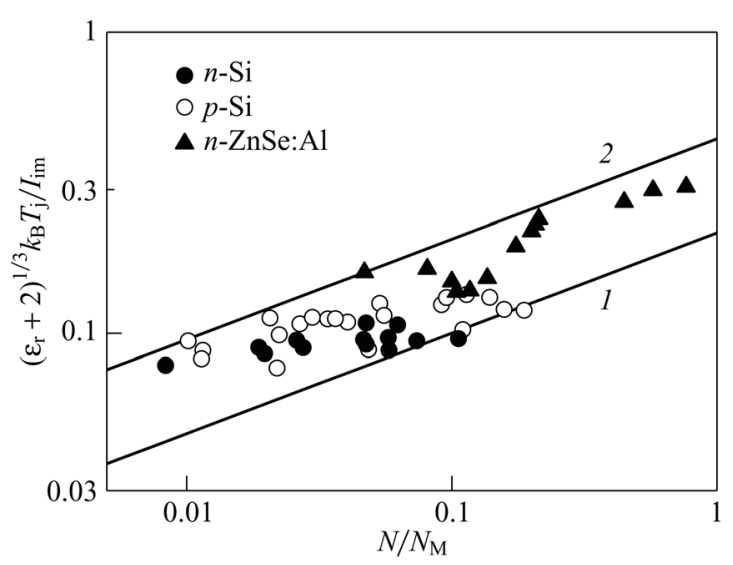

FIG. 4. Dependence on concentration of the doping impurity $N$ of the transition temperature $T_{\mathrm{j}}$ from hopping to the band conduction in $n$-Si, $p$-Si, and $n$-ZnSe:Al. The lines are calculations by using Eq. (7) for $K=0.02$ (1) and $K=0.15$ (2). The points are experimental data from the works: Refs. 28-30 $(n-\mathrm{Si}, p-\mathrm{Si})$ and Ref. 31 (n-ZnSe).

diamond of $p$-type ${ }^{18-24}$ ( $p$-Dia:B) and gallium arsenide of $n$-type $\mathrm{e}^{25-27}(n-\mathrm{GaAs})$ on the concentration of majority (doping) impurity. In order to compare $T_{\mathrm{j}}$ for materials with different relative permittivity $\varepsilon_{\mathrm{r}}$ in the same plot, we, in accordance with relation (7), carried over the multiplier $\left(\varepsilon_{\mathrm{r}}+2\right)^{1 / 3}$ into the left part. Parameters of semiconductors $\left(\varepsilon_{\mathrm{r}}, I_{\mathrm{im}}, N_{\mathrm{M}}\right)$ used for the construction of experimental data dependences are given in Table I. Figure 4 shows the concentration dependence of $T_{\mathrm{j}}$ for silicon crystals of $n$ - and -types $^{28-30}(n-\mathrm{Si}, p$-Si) and for aluminum-doped zinc selenide crystals ${ }^{31}$ (n-ZnSe:Al). It is seen from Figs. 3 and 4 that the calculations of $T_{\mathrm{j}}$ agree with experimental data in the limits of typical deviations of compensation ratios $K$ for these semiconductor materials. Note that, for $n$-type semiconductor samples shown in Figs. 3 and 4, only the $D$-bot donor band is pronounced, i.e., donors are in the charge states $(0)$ and $(+1)$.

\section{SCREENING LENGTH OF ELECTROSTATIC FIELD IN THE DEBYE-HÜCKEL APPROXIMATION AT THE NEAREST NEIGHBOR HOPPING CONDUCTION}

Let us consider implementation of characteristic temperatures $T_{\mathrm{j}}$ and $T_{\mathrm{h}} \approx T_{\mathrm{j}} / 3$ for estimates of concentration of hydrogen-like impurities from measurements of capacitance in a metal-insulator-semiconductor (MIS) structure on the example of the crystalline semiconductor of $p$-type (Fig. 1).

When the band conduction dominates $\left(\sigma_{p} \gg \sigma_{\mathrm{h}}\right)$, the capacitance of a MIS structure in the regime of the flat $c$-and v-bands is (see, e.g., Refs. 2 and 62) $C_{\mathrm{fb}}=C_{\mathrm{ox}} C_{\mathrm{DH}} /$ $\left(C_{\mathrm{ox}}+C_{\mathrm{DH}}\right)$, where $C_{\mathrm{ox}}=\varepsilon_{\mathrm{ox}} / d_{\mathrm{ox}}$ is the capacitance of an insulator with permittivity $\varepsilon_{\mathrm{ox}}$ and thickness $d_{\mathrm{ox}}$ between a metal electrode of the unit area and semiconductor, and $C_{\mathrm{DH}}=\varepsilon_{\mathrm{r}} \varepsilon_{0} / \Lambda_{p}$ is the capacitance of the semiconductor with permittivity $\varepsilon=\varepsilon_{\mathrm{r}} \varepsilon_{0}$ and the screening length $\Lambda_{p}$. The capacitance of insulator $C_{\mathrm{ox}}$ can be quite easily and accurately measured. ${ }^{63}$ Then, the screening length of an external electrostatic field (screened by holes of the $v$-band), after Debye-Hückel, $\Lambda_{p}=\left(\varepsilon_{\mathrm{r}} \varepsilon_{0} k_{\mathrm{B}} T / e^{2} p\right)^{1 / 2}$ is found from the flatband quasistatic capacitance of a MIS structure $C_{\mathrm{fb}}$. Thus, this procedure allows to find concentration of holes $p$ from $\Lambda_{p}$ (when acceptors are totally ionized $\left.p=(1-K) N\right)$.

When the hopping conduction by holes via acceptors dominates $\left(\sigma_{\mathrm{h}} \gg \sigma_{p}\right)$, to the best of our knowledge, the possibility to determine the electrostatic field screening length $\Lambda_{\mathrm{h}}$ from measurements of quasistatic capacitance $C_{\mathrm{DH}}=$ $\varepsilon_{\mathrm{r}} \varepsilon_{0} / \Lambda_{\mathrm{h}}$ is not realized yet.

Hopping conduction in the $\mathrm{NNH}$ regime dominates $\left(\sigma_{\mathrm{h}} \gg \sigma_{p}\right.$; Fig. 1) in the vicinity of temperature $T_{\mathrm{h}}=T_{\mathrm{j}} / 3$, where $T_{\mathrm{j}}$ is defined by formula (5). The screening length determined by holes hopping between acceptors has, in the Debye-Hückel approximation, the form ${ }^{39}$

$$
\Lambda_{\mathrm{h}}=\left(\frac{\varepsilon_{\mathrm{r}} \varepsilon_{0} \xi_{\mathrm{h}} k_{\mathrm{B}} T_{\mathrm{h}}}{e^{2} N_{\mathrm{h}}}\right)^{1 / 2}
$$

where $N_{\mathrm{h}}=(1-K) K N$ is the effective concentration of holes hopping between acceptors in the charge states $(0)$ and $(-1)$. The parameter $\xi_{\mathrm{h}} \geq 1$ characterizes the influence of fluctuations of the electrostatic potential in a crystal on the ratio of the hopping diffusion coefficient $D_{\mathrm{h}}$ to the drift hopping mobility $M_{\mathrm{h}}$ of holes, $D_{\mathrm{h}} / M_{\mathrm{h}}=\xi_{\mathrm{h}} k_{\mathrm{B}} T / e^{39,40}$ When the acceptor $A$-bot bandwidth $W$ (see Fig. 1) is less or about thermal energy $k_{\mathrm{B}} T_{\mathrm{h}}$, then $\xi_{\mathrm{h}} \approx 1$. . $^{39,40,64}$

At hopping conduction the root-mean-square electrostatic fluctuation of acceptor energy levels (the width $W$ of the acceptor $A$-bot band), at a random distribution of impurity ions over a crystalline semiconductor and taking into account the Coulomb interaction with the nearest neighbor ions only, has the form ${ }^{10,65}$

$$
\begin{aligned}
W & =\left(\sum_{i=1}^{2} \int_{0}^{\infty} P_{i} U_{i}^{2} \mathrm{~d} r\right)^{1 / 2} \\
& \approx 1.637 \frac{e^{2}}{4 \pi \varepsilon_{\mathrm{r}} \varepsilon_{0}}\left(\frac{8 \pi}{3} K N\right)^{1 / 3}
\end{aligned}
$$

where $P_{i} \mathrm{~d} r=4 \pi r^{2} C_{i} \exp \left[-2 K N\left(4 \pi r^{3} / 3\right)\right] \mathrm{d} r$ is the Poisson probability that the nearest to a given ion another ion of type $i$ (acceptor $(i=1)$ or donor $(i=2))$ is positioned at the distance from $r$ to $r+\mathrm{d} r$ (see Refs. 44 and 45) and there are no other impurity ions in a sphere of the volume $4 \pi r^{3} / 3$ centered at a given ion, $\sum_{i=1}^{2} C_{i}=2 K N$ is the concentration of all ions in a crystal $\left(C_{1}=C_{2}=K N\right), U_{i}(r)= \pm e^{2} / 4 \pi \varepsilon_{\mathrm{r}} \varepsilon_{0} r$ is the Coulomb energy of interaction between the nearest neighbor ions having the same $(\operatorname{sign}+)$ or different $(\operatorname{sign}-)$ charge states, $\sum_{i=1}^{2} \int_{0}^{\infty} P_{i} U_{i} \mathrm{~d} r=0$ follows from the electrical neutrality of a crystal $\left(N_{-1}=N_{+1}=K N\right), \sum_{i=1}^{2} P_{i}$ is the probability density of the nearest neighbor impurity ions, and $\int_{0}^{\infty} \sum_{i=1}^{2} P_{i} \mathrm{~d} r=1$. Further, we assume that, for moderately doped $\left(0.01<N / N_{\mathrm{M}}<0.5\right)$ and moderately compensated $(0.1<K<0.9)$ semiconductors, the root-mean-square fluctuation of acceptor energy levels by Eq. (9) is much larger than the quantum-mechanical splitting of levels due to finiteness of localization time of hopping holes (see estimations in Ref. 64). Note that the quantum-mechanical splitting of levels is more noticeable for the $A$-top band than for the $A$-bot band (see Fig. 1). 
Note that, at temperature $T_{\mathrm{j}}$, the concentration of holes is $p \ll K(1-K) N$, leading to the root-mean-square fluctuation of the potential energy of a hole in the valence band $W_{p} \ll W$ (see Ref. 66 and references therein). Due to the mobility edge $\left(\left|E_{v}^{(\mu)}\right| \approx W_{p}\right)$ is close to the top of the v-band (see Fig. 1), the fluctuations of the top of the valence band can be neglected $\left(W_{p} \ll k_{\mathrm{B}} T_{\mathrm{j}}\right.$ ) for $T_{\mathrm{j}}$ calculations. Thus, the formula (5) for $T_{\mathrm{j}}$ is quite correct. At temperature $T_{\mathrm{h}}=T_{\mathrm{j}} / 3$, where the hopping conduction dominates $\left(\sigma_{\mathrm{h}} \gg \sigma_{p}\right)$, the ratio of the acceptor bandwidth $W$ by Eq. (9) to the thermal energy $k_{\mathrm{B}} T_{\mathrm{h}}$ is $W / k_{\mathrm{B}} T_{\mathrm{h}} \approx 13.7$ and it does not depend on the acceptor concentration $N$ and the compensation ratio $K$. Thus, it follows from Eqs. (5) and (9) that $W \gg k_{\mathrm{B}} T_{\mathrm{h}}$.

By substituting temperature $T_{\mathrm{h}}=T_{\mathrm{j}} / 3$ according to Eq. (5) into the expression (8) for the screening length $\Lambda_{\mathrm{h}}$, we obtain

$$
\Lambda_{\mathrm{h}} \approx \frac{0.14}{(K N)^{1 / 3}}\left(\frac{\xi_{\mathrm{h}}}{1-K}\right)^{1 / 2},
$$

where $\xi_{\mathrm{h}} \approx K(1-K)\left(W / k_{\mathrm{B}} T_{\mathrm{h}}\right) \sqrt{2 \pi} \exp \left(\chi^{2}\right)$ for $W \gg k_{\mathrm{B}} T_{\mathrm{h}}$, assuming the Gaussian distribution of acceptor energy levels (line $g_{\mathrm{b}}$ in Fig. 1), and $\chi$ is the parameter obtained from the electrical neutrality condition of a crystal: $2 K=1-\operatorname{erf}(\chi)$; $\xi_{\mathrm{h}}=1$ for $W \ll k_{\mathrm{B}} T_{\mathrm{h}}$. (Derivation of expressions for $\xi_{\mathrm{h}}$ and $\chi$ is presented in Refs. 39, 40, and 64.) Note that the screening length $\Lambda_{\mathrm{h}}$ given by Eq. (10) for $W \gg k_{\mathrm{B}} T_{\mathrm{h}}$ is larger than an average distance between neighbor impurity atoms ${ }^{45}$ $d_{\mathrm{im}} \approx 0.554 /[(1+K) N]^{1 / 3}$ for all values of the compensation ratio $K$ (see Fig. 5). As far as $\Lambda_{\mathrm{h}}>d_{\mathrm{im}}$, we can state that, according to Ref. 67, the Debye-Hückel approximation at temperature $T_{\mathrm{h}}$ for the wide impurity band $\left(W \gg k_{\mathrm{B}} T_{\mathrm{h}}\right.$, $\left.\xi_{\mathrm{h}}>1\right)$ is valid. To the contrary, for the narrow impurity band $\left(W \ll k_{\mathrm{B}} T_{\mathrm{h}}, \xi_{\mathrm{h}}=1\right)$, we get $d_{\mathrm{im}}>\Lambda_{\mathrm{h}}$ for $0.1<K<0.9$. Thus, the Debye-Hückel approximation for screening length calculation in the case of the narrow impurity band and intermediate compensation ratios is not valid.

From formula (10), for the wide impurity band $\left(W \gg k_{\mathrm{B}} T_{\mathrm{h}}, \xi_{\mathrm{h}}>1\right)$, it follows that the quantity $\Lambda_{\mathrm{h}} N^{1 / 3} \approx$ $0.81 K^{1 / 6} \exp \left(\chi^{2} / 2\right)$, where $\operatorname{erf}(\chi)=1-2 K$, depends only on the compensation ratio (see Fig. 5, curve 1). The dashed line

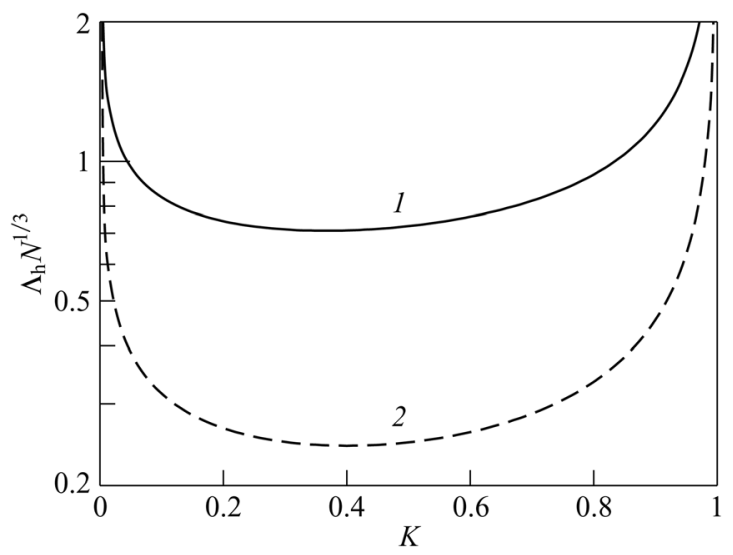

FIG. 5. Dependence of the screening length $\Lambda_{\mathrm{h}}$ at hopping electrical conduction via hydrogen-like impurities on the compensation ratio $K$ calculated using Eq. (10) for temperature $T_{\mathrm{h}}=T_{\mathrm{j}} / 3$ : the curve 1 -at $\xi_{\mathrm{h}}>1\left(W \gg k_{\mathrm{B}} T_{\mathrm{h}}\right)$ and the curve 2 -at $\xi_{\mathrm{h}}=1\left(W \ll k_{\mathrm{B}} T_{\mathrm{h}}\right)$. in Fig. 5 shows calculations of the screening length $\Lambda_{\mathrm{h}}$ by models ${ }^{68,69}$ for the case of the narrow impurity band $\left(W \ll k_{\mathrm{B}} T_{\mathrm{h}}, \xi_{\mathrm{h}}=1\right)$ at temperature $T_{\mathrm{h}}=T_{\mathrm{j}} / 3$. It is seen in Fig. 5 that the screening length $\Lambda_{\mathrm{h}} \propto N^{-1 / 3}$ at temperature $T_{\mathrm{h}}$ weakly depends on the compensation ratio (for intermediate values of $K$ ) for both the wide (curve 1 ) and narrow (curve 2 ) impurity bands. Unlimited increase of the screening length at very low $(K \rightarrow 0)$ and very high $(K \rightarrow 1)$ compensation ratios is related to diminishing of the effective concentration of holes hopping between acceptors $N_{\mathrm{h}}=(1-K) K N \rightarrow 0$.

Note that, at a large degree of compensation $1-K \ll 1$, the long-range fluctuations of electrostatic potential energy of impurity ions arise (with characteristic size much larger than the average distance between impurity ions). ${ }^{6,70}$ Thus, for very high compensation ratios, the expression Eq. (10) becomes qualitative, due to Eq. (9) describes only shortrange electrostatic fluctuations. To be more precise, Eq. (9) takes into account only Coulomb interactions between randomly distributed nearest neighbor impurity ions in a semiconductor.

\section{CONCLUSIONS}

In the paper, we employ the virial theorem for a single typical pair "an electron in the $c$-band - a nearest to it donor in the charge state $(+1)$ " for nondegenerate semiconductor of $n$-type or a pair "a hole in the $v$-band - a nearest to it acceptor in the charge state $(-1)$ " for a nondegenerate semiconductor of $p$-type. As a result, an expression is obtained for the transition temperature $T_{\mathrm{j}}$ from the band electrical conduction (in the $c$ - or $v$-band) to the electrical conduction by hops of electrons or holes between hydrogen-like impurities. Temperature $T_{\mathrm{j}}$ increases with the concentration of the doping impurity as well as with the compensation ratio. Calculated values of $T_{\mathrm{j}}$ agree well with known experimental data. For temperature $T_{\mathrm{h}}=T_{\mathrm{j}} / 3$, we calculate the screening length $\Lambda_{\mathrm{h}}$ (after Debye-Hückel) when the hopping conduction dominates in approximations of the wide and narrow impurity bands. The value of $\Lambda_{\mathrm{h}}$ can be found from the measurements of quasistatic capacitance $C_{\mathrm{DH}}=\varepsilon_{\mathrm{r}} \varepsilon_{0} / \Lambda_{\mathrm{h}}$ (accounted for the unit area surface of semiconductor included in a MIS structure). Using the value of $\Lambda_{\mathrm{h}}$, the concentration $N$ (when $K$ is known) or compensation $K$ (when $N$ is known) can be found within the framework of our model (for the wide impurity band, where the Debye-Hückel approximation is applicable).

\section{ACKNOWLEDGMENTS}

The work was supported by Belarusian scientific program "Crystal and molecular structures" and Russian foundation for basic research (Grant No. 10-02-00629).

\footnotetext{
${ }^{1}$ A. Rogalski and F. Sizov, Opto-Electron. Rev. 19, 346 (2011).

${ }^{2} \mathrm{~K}$. K. Ng, Complete Guide to Semiconductor Devices (Wiley, New York, 2002), Chaps. 63 and 20.

${ }^{3}$ N. A. Poklonski, S. A. Vyrko, and A. G. Zabrodskii, Semiconductors 40, 394 (2006); Fiz. Tekh. Poluprovodn. 40, 400 (2006).

${ }^{4}$ S. Datta, Phys. Rev. Lett. 44, 828 (1980).

${ }^{5}$ I. P. Zvyagin, Phys. Status Solidi B 101, 9 (1980).

${ }^{6}$ B. I. Shklovskii and A. L. Efros, Electronic Properties of Doped Semiconductors (Springer, Berlin, 1984).

${ }^{7}$ M. Pollak, Phys. Status Solidi B 230, 295 (2002).
} 
${ }^{8}$ V. F. Gantmakher and V. T. Dolgopolov, Phys. Usp. 51, 3 (2008); Usp. Fiz. Nauk 178, 3 (2008).

${ }^{9}$ A. G. Zabrodskii, A. G. Andreev, and M. V. Alekseenko, Sov. Phys. Semicond. 26, 244 (1992); Fiz. Tekh. Poluprovodn. 26, 431 (1992).

${ }^{10}$ N. A. Poklonskii, A. I. Syaglo, and G. Biskupski, Semiconductors 33, 402 (1999); Fiz. Tekh. Poluprovodn. 33, 415 (1999).

${ }^{11}$ A. G. Andreev, S. V. Egorov, A. G. Zabrodskii, R. V. Parfen'ev, and A. V. Chernyaev, Semiconductors 34, 768 (2000); Fiz. Tekh. Poluprovodn. 34, 796 (2000).

${ }^{12}$ H. Fritzsche and M. Cuevas, Phys. Rev. 119, 1238 (1960).

${ }^{13} \mathrm{H}$. Fritzsche and M. Cuevas, in Proc. of International Conference on Semiconductor Physics, Prague, 1960 (Academy of Sciences of the Czech Republic, Prague, 1961), pp. 222-224.

${ }^{14}$ H. C. Thomas and B. Covington, J. Appl. Phys. 48, 3434 (1977).

${ }^{15}$ L. V. Govor, V. P. Dobrego, and N. A. Poklonskii, Sov. Phys. Semicond. 18, 1292 (1984); Fiz. Tekh. Poluprovodn. 18, 2075 (1984).

${ }^{16}$ A. G. Andreev, V. V. Voronkov, G. I. Voronkova, A. G. Zabrodskii, and E. A. Petrova, Semiconductors 29, 1162 (1995); Fiz. Tekh. Poluprovodn. 29, 2218 (1995).

${ }^{17}$ A. G. Zabrodskii, A. G. Andreev, and S. V. Egorov, Phys. Status Solidi B 205, 61 (1998).

${ }^{18}$ O. I. Smirnova, E. E. Godik, and A. G. Gontar', Sov. Phys. Semicond. 19, 398 (1985); Fiz. Tekh. Poluprovodn. 19, 646 (1985).

${ }^{19}$ J. Mort, K. Okumura, and M. Machonkin, Philos. Mag. B 63, 1031 (1991).

${ }^{20}$ H. Shiomi, Y. Nishibayashi, and N. Fujimori, Jpn. J. Appl. Phys. 30, 1363 (1991).

${ }^{21}$ E. P. Visser, G. J. Bauhuis, G. Janssen, W. Vollenberg, W. J. P. van Enckevort, and L. J. Giling, J. Phys.: Condens. Matter 4, 7365 (1992).

${ }^{22}$ D. M. Malta, J. A. von Windheim, H. A. Wynands, and B. A. Fox, J. Appl. Phys. 77, 1536 (1995).

${ }^{23}$ T. H. Borst and O. Weis, Phys. Status Solidi A 154, 423 (1996)

${ }^{24} \mathrm{~V}$. Mortet, M. Daenen, T. Teraji, A. Lazea, V. Vorlicek, J. D'Haen, K. Haenen, and M. D'Olieslaeger, Diamond Relat. Mater. 17, 1330 (2008),

${ }^{25}$ D. Lemoine, C. Pelletier, S. Rolland, and R. Granger, Phys. Lett. A 56, 493 (1976).

${ }^{26}$ O. V. Emel'yanenko, T. S. Lagunova, D. N. Nasledov, A. A. Telegin, and Z. I. Chugueva, Sov. Phys. Semicond. 10, 759 (1976); Fiz. Tekh. Poluprovodn. 10, 1280 (1976).

${ }^{27}$ M. Benzaquen and D. Walsh, Phys. Rev. B 30, 7287 (1984).

${ }^{28}$ R. K. Ray and H. Y. Fan, Phys. Rev. 121, 768 (1961).

${ }^{29}$ K. R. Atkins, R. Donovan, and R. H. Walmsley, Phys. Rev. 118, 411 (1960).

${ }^{30}$ G. A. Swartz, J. Phys. Chem. Solids 12, 245 (1960).

${ }^{31}$ D. D. Nedeoglo and V. A. Kasiyan, Phys. Status Solidi B 210, 301 (1998).

${ }^{32}$ E. M. Gershenzon, F. M. Ismagilova, and L. B. Litvak-Gorskaya, Semiconductors 28, 401 (1994); Fiz. Tekh. Poluprovodn. 28, 671 (1994).

${ }^{33}$ N. F. Mott, Philos. Mag. 19, 835 (1969).

${ }^{34}$ A. L. Efros and B. I. Shklovskii, J. Phys. C 8, L49 (1975).

${ }^{35}$ V. D. Kagan, Phys. Solid State 42, 824 (2000); Fiz. Tverd. Tela (Leningrad) 42, 805 (2000).

${ }^{36}$ A. G. Zabrodskii, Phil. Mag. B 81, 1131 (2001).

${ }^{37}$ Ya. E. Pokrovskii, O. I. Smirnova, and N. A. Khvalkovskii, J. Exp. Theor. Phys. 85, 121 (1997); Zh. Eksp. Teor. Fiz. 112, 221 (1997).

${ }^{38}$ J. S. Blakemore, Semiconductor Statistics (Dover, New York, 2002), Chap. 3.

${ }^{39}$ N. A. Poklonski and V. F. Stelmakh, Phys. Status Solidi B 117, 93 (1983).

${ }^{40}$ N. A. Poklonski, S. A. Vyrko, and A. G. Zabrodskii, Solid State Commun. 149, 1248 (2009).
${ }^{41}$ H. Goldstein, C. P. Poole, and J. L. Safko, Classical Mechanics (AddisonWesley, Reading, MA, 2002), Chap. 3.

${ }^{42}$ B. V. Vasil'ev and V. L. Lyuboshits, Phys. Usp. 37, 345 (1994); Usp. Fiz. Nauk 164, 367 (1994).

${ }^{43}$ T. Koga, Introduction to Kinetic Theory: Stochastic Processes in Gaseous Systems (Pergamon, Oxford, 1970), Chap. 4, 9, and Appendix G.

${ }^{44}$ S. Chandrasekhar, Rev. Mod. Phys. 15, 1 (1943).

${ }^{45}$ P. Whittle, Probability (Penguin, Cambridge, 1970), Chap. 4.

${ }^{46}$ W. Ebeling, W. D. Kraeft, and D. Kremp, Theory of Bound States and Ionization Equilibrium in Plasmas and Solids (Akademie-Verlag, Berlin, 1976).

${ }^{47}$ B. V. Alekseev, Phys. Usp. 46, 139 (2003); Usp. Fiz. Nauk 173, 145 (2003).

${ }^{48}$ N. F. Mott, Metal-Insulator Transitions (Taylor and Francis, London, 1990).

${ }^{49}$ N. A. Poklonski, S. A. Vyrko, and A. G. Zabrodskii, Phys. Solid State 46, 1101 (2004); Fiz. Tverd. Tela (Leningrad) 46, 1071 (2004).

${ }^{50}$ A. G. Zabrodskii and M. V. Alekseenko, Semiconductors 28, 101 (1994); Fiz. Tekh. Poluprovodn. 28, 168 (1994).

${ }^{51}$ I. S. Shlimak, Phys. Solid State 41, 716 (1999); Fiz. Tverd. Tela (Leningrad) 41, 794 (1999).

${ }^{52}$ Semiconductors: Data Handbook, edited by O. Madelung (Springer, Berlin, 2004).

${ }^{53}$ R. Rentzsch, K. J. Friedland, and A. N. Ionov, Phys. Status Solidi B 146, 199 (1988).

${ }^{54}$ T. I. Voronina, A. N. Dakhno, O. V. Emel'yanenko, T. S. Lagunova, and S. P. Starosel'tseva, Sov. Phys. Semicond. 22, 779 (1988); Fiz. Tekh. Poluprovodn. 22, 1230 (1988)

${ }^{55}$ A. P. Long and M. Pepper, J. Phys. C 17, L425 (1984).

${ }^{56}$ U. Thomanschefsky and D. F. Holcomb, Phys. Rev. B 45, 13356 (1992).

${ }^{57}$ P. Dai, Y. Zhang, and M. P. Sarachik, Phys. Rev. B 49, 14039 (1994).

${ }^{58}$ P. F. Newman and D. F. Holcomb, Phys. Rev. B 28, 638 (1983).

${ }^{59}$ P. Dai, S. Bogdanovich, Y. Zhang, and M. P. Sarachik, Phys. Rev. B 52, 12439 (1995).

${ }^{60}$ Neutron Transmutation Doping of Semiconductor Materials, edited by R. D. Larrabee (Plenum, New York, 1984).

${ }^{61}$ A. G. Zabrodskii and M. V. Alekseenko, in Proc. of $23 \mathrm{rd}$ International Conference on the Physics of Semiconductors, Berlin, Germany, 21-26 July 1996, edited by M. Scheffler and R. Zimmermann (World Scientific, Singapore, 1996), Vol. 4, pp. 2681-2684.

${ }^{62}$ S. M. Sze and K. K. Ng, Physics of Semiconductor Devices (Wiley, New York, 2007), Chap. 4.

${ }^{63}$ A. G. Zhdan, N. F. Kukharskaya, and G. V. Chucheva, Instrum. Exp. Tech. 47, 791 (2004); Prib. Tekh. Eksp. 6, 77 (2004).

${ }^{64}$ N. A. Poklonski, S. A. Vyrko, and A. G. Zabrodskii, Semicond. Sci. Technol. 25, 085006 (2010).

${ }^{65}$ N. A. Poklonskii, A. I. Syaglo, and F. N. Borovik, Semiconductors 30, 924 (1996); Fiz. Tekh. Poluprovodn. 30, 1767 (1996).

${ }^{66}$ N. A. Poklonski, S. A. Vyrko, O. N. Poklonskaya, and A. G. Zabrodskii, Phys. Status Solidi B 246, 158 (2009).

${ }^{67}$ D. ter Haar, "The plasma," in Problems in Thermodynamics and Statistical Physics, edited by P. T. Landsberg (Pion, London, 1971), Chap. 14, pp. 333-340.

${ }^{68}$ N. A. Poklonskii, V. F. Stelmakh, V. D. Tkachev, and S. V. Voitikov, Phys. Status Solidi B 88, K165 (1978).

${ }^{69}$ A. A. Uzakov and A. L. Efros, Sov. Phys. JETP 54, 1028 (1981); Zh. Eksp. Teor. Fiz. 81, 1940 (1981).

${ }^{70}$ Yu. Ya. Tkach, Sov. Phys. JETP 74, 109 (1992); Zh. Eksp. Teor. Fiz. 101, 203 (1992). 